\title{
The Signed Domination Number of Cartesian Product of Two Paths
}

\author{
Mohammad Hassan, Muhsin Al Hassan, Mazen Mostafa \\ Department of Mathematics, Faculty of Science, Tishreen University, Lattakia, Syria \\ Email: sf-qa u@tishreen.edu.sy,mmuuhhssiinn@gmail.com, mazenmostafa1979@gmail.com
}

How to cite this paper: Hassan, M., Al Hassan, M. and Mostafa, M. (2020) The Signed Domination Number of Cartesian Product of Two Paths. Open Journal of Discrete Mathematics, 10, 45-55. https://doi.org/10.4236/ojdm.2020.102005

Received: November 26, 2019

Accepted: January 19, 2020

Published: January 22, 2020

Copyright $\odot 2020$ by author(s) and Scientific Research Publishing Inc. This work is licensed under the Creative Commons Attribution International License (CC BY 4.0).

http://creativecommons.org/licenses/by/4.0/

\begin{abstract}
Let $G$ be a finite connected simple graph with vertex set $V(G)$ and edge set $E(G)$. A function $f: V(G) \rightarrow\{-1,1\}$ is a signed dominating function if for every vertex $v \in V(G)$, the closed neighborhood of $v$ contains more vertices with function values 1 than with -1 . The signed domination number $\gamma_{s}(G)$ of $G$ is the minimum weight of a signed dominating function on $G$. In this paper, we calculate The signed domination numbers of the Cartesian product of two paths $P_{m}$ and $P_{n}$ for $m=3,4,5$ and arbitrary $n$.
\end{abstract}

\section{Keywords}

Path, Cartesian Product, Signed Dominating Function, Signed Domination Number

\section{Introduction}

Let $G$ be a finite simple connected graph with vertex set $V(G)$ and edge set $E(G)$. The neighborhood of $v$, denoted $N(v)$, is set $\{u: u v \in E(G)\}$ and the closed neighborhood of $v$, denoted $N[v]$, is set $N(v) \cup\{v\}$. The function $f$ is a signed dominating function if for every vertex $v \in V$, the closed neighborhood of $v$ contains more vertices with function value 1 than with -1 . The signed domination number of $G, \gamma_{s}(G)$, is the minimum weight of a signed dominating function on $G$.

In [1], Dunbar et al. introduced this concept and it has been studied by several authors [1] [2] [3] [4], in [5] Haas and Wexler had found the signed domination number of $P_{2} \times P_{n}$ and $P_{2} \times C_{n}$. In [6] Hosseini gave a lower and upper bound for the signed domination number for any graph.

We consider when we represent the $P_{m} \times P_{n}$ graph to find the signed dominating function that the black circles refer to the graph vertices which weight 1 , 
and the white circles refer to the graph vertices which weight -1 . Let $f$ be a signed dominating function of the $P_{m} \times P_{n}$ graph and, $A=\{v \in V: f(v)=1\}$, $B=\{v \in V: f(v)=-1\}$, then $|A|+|B|=m \cdot n$, is number of the graph vertices, and $\gamma_{s}\left(P_{m} \times P_{n}\right)=m \cdot n-2|B|=|A|-|B|$. Let $K_{j}$ the $f^{\text {th }}$ column vertices, and also $A_{i}=\left\{v \in K_{i}: f(v)=1\right\}, B_{i}=\left\{v \in K_{i}: f(v)=-1\right\}$ then $\left|A_{j}\right|+\left|B_{j}\right|=m$.

\section{Main Results}

In this paper we will show three theorems to find the signed domination number of Cartesian product of $P_{m} \times P_{n}$.

Theorem 2.1. Let $n$ be a positive integer:

If $n \equiv 0(\bmod 3)$, then $\gamma_{s}\left(P_{3} \times P_{n}\right)=\frac{5 n}{3}$;

If $n \equiv 1(\bmod 3)$, then $\gamma_{s}\left(P_{3} \times P_{n}\right)=\frac{5(n-1)}{3}+1$;

If $n \equiv 2(\bmod 3)$, then $\gamma_{s}\left(P_{3} \times P_{n}\right)=\frac{5(n-2)}{3}+2$.

Proof: Case $n \equiv 0(\bmod 3)$

Let $f$ be a signed dominating function of $\left(P_{3} \times P_{n}\right)$, then for any $j$ were $2 \leq j \leq$ $n-1$, then $\sum_{k=i-1}^{j+1}\left|B_{K}\right| \leq 2$. We discuss the following cases:

Case a. $\left|B_{j}\right|=2$ (Figure 1)

We notices that the first and last columns can't include more than one vertex of the $B$ set vertices. But in the case $2 \leq j \leq n-1$ and $\left|B_{j}\right|=2$, the vertices $(1, j)$ and $(3, j)$ belong to the $B$ set vertices and all the $K_{j+1^{\text {th }}}, K_{j-1^{\text {th }}}$ vertices belong to the $A$ set.

Case b. $\left|B_{j}\right|=1$ (Figure 2)

We discuss the following cases:

b.1. If $(1, j) \in B$ then all the vertices $(1, j-1),(2, j-1),(1, j+1)$ and $(2, j+$ 1 ) belong to the $A$ set, and one of the vertices $(3, j-1)$ or $(3, j+1)$ at most can belong to the $B$ set vertices.

b.2. If $(2, j) \in B$ then all the vertices $(1, j-1),(3, j-1),(1, j+1)$ and $(3, j+$ 1 ) belong to the $A$ set, and one vertex of the vertices $(2, j-1)$ or $(2, j+1)$ at most belong to the $B$ set vertices.

b.3. If $(3, j) \in B$ then all the vertices $(2, j-1),(3, j-1),(2, j+1)$ and $(3, j+$ $1)$ belong to the $A$ set, and one of the vertices $(1, j-1)$ or $(1, j+1)$ at most belong to the $B$ set vertices.

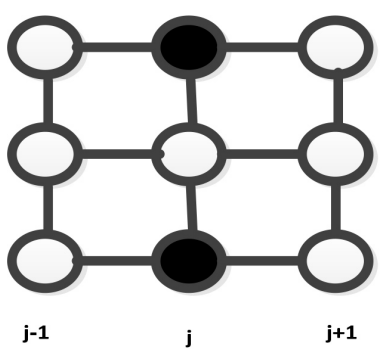

Figure 1. Case a. 


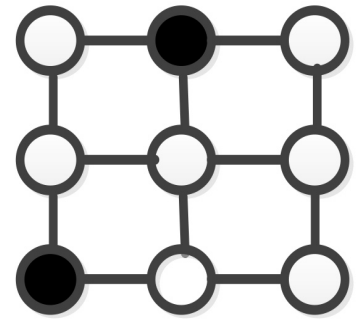

j-1

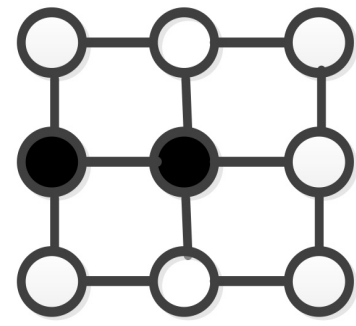

j-1

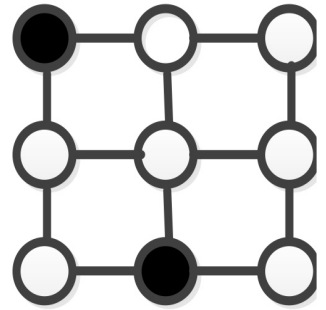

j-1

j+1

Figure 2. Case b.

Case c. $\left|B_{j}\right|=0$ (Figure 3)

When the $f^{\text {th }}$ column doesn't include any one of the $B$ set vertices, it is possible that the vertices $(1, j+1)$ and $(3, j+1)$ belong to the $B$ set provided that the $j+$ $1^{\text {th }}$ column isn't the last column then all the $j+2^{\text {th }}$ column vertices belong to the $A$ set, or the tow vertices $(1, j-1)$ and $(3, j-1)$ belong to the $B$ set provided that the $j-1^{\text {th }}$ column isn't the first one, and all the $j-2^{\text {th }}$ column vertices belong to the $A$ set.

Whereas the $j+1^{\text {th }}$ column includes one of the $B$ set vertices, then the $j+2^{\text {th }}$ column will include one of the $B$ set vertices at most. Also if the $j-1^{\text {th }}$ column includes one of the $B$ set then the $j-2$ will include one of the $B$ set vertices at most.

We conclude from the previous cases that if $2 \leq j \leq n-1$, then $\sum_{k=i-1}^{j+1}\left|B_{K}\right| \leq 2$. And all three successive columns include two vertices at most weighted with -1 , so seven vertices at least is weighted the weight 1 , consequently:

$$
\gamma_{s}\left(P_{3} \times P_{n}\right) \geq \frac{5 n}{3}: n \equiv 0(\bmod 3)
$$

To find the upper bound of the signed domination number of $\left(P_{3} \times P_{n}\right)$ graph, Let's define $B=\left\{(0,3 j): 0 \leq j \leq\left\lfloor\frac{n-1}{3}\right\rfloor \cup(2,3 j+1): 0 \leq j \leq\left\lfloor\frac{n-2}{3}\right\rfloor\right\}$ (Figure 4).

If $B$ is the previously defined set and represents the vertices have the weight -1 , then every one of the $P_{3} \times P_{n}$ graph vertices achieves the signed dominating function, and $|B| \geq \frac{2 n}{3}$ then: $\gamma_{s}\left(P_{3} \times P_{n}\right) \leq 3 n-2\left(\frac{2 n}{3}\right) \leq \frac{5 n}{3}$.

Consequently: $\gamma\left(P_{3} \times P_{n}\right)=\frac{5 n}{3}: n \equiv 0(\bmod 3)$.

Case $n \equiv 1(\bmod 3)($ Figure 5$)$

If we add a column to the previous graph in case $[n \equiv 0(\bmod 3)]$ then one vertex at most can have the weight -1 , so, when we add that vertex to the $B$ set this makes $f$ a signed dominating function, so:

$$
B=\left\{(0,3 j): 0 \leq j \leq\left\lfloor\frac{n-1}{3}\right\rfloor \cup(2,3 j+1): 0 \leq j \leq\left\lfloor\frac{n-2}{3}\right\rfloor\right\} \cup\{(n, 0)\} .
$$

Consequently: $\gamma_{s}\left(P_{3} \times P_{n}\right)=\frac{5(n-1)}{3}+1$. 


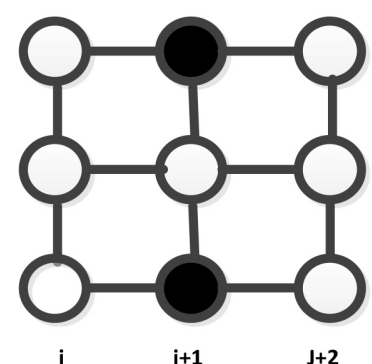

j

3.

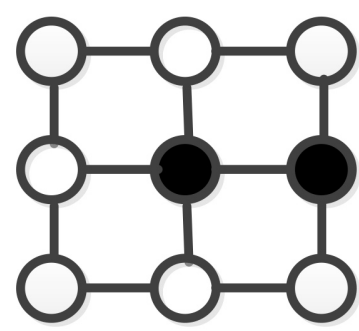

j+1

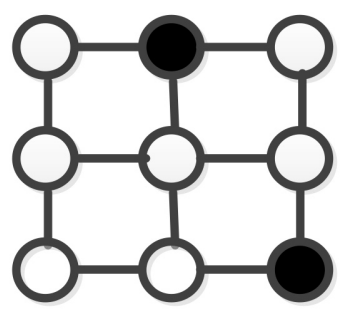

j $\quad$ j+1 $\quad$ j+2

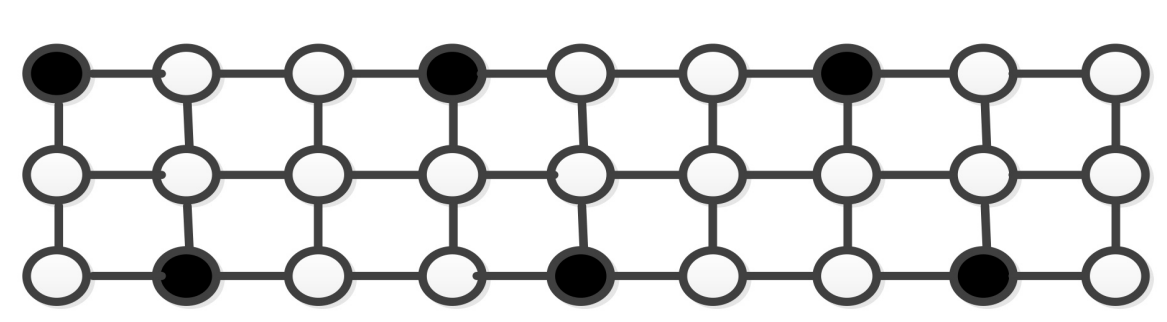

Figure 4. The B set.
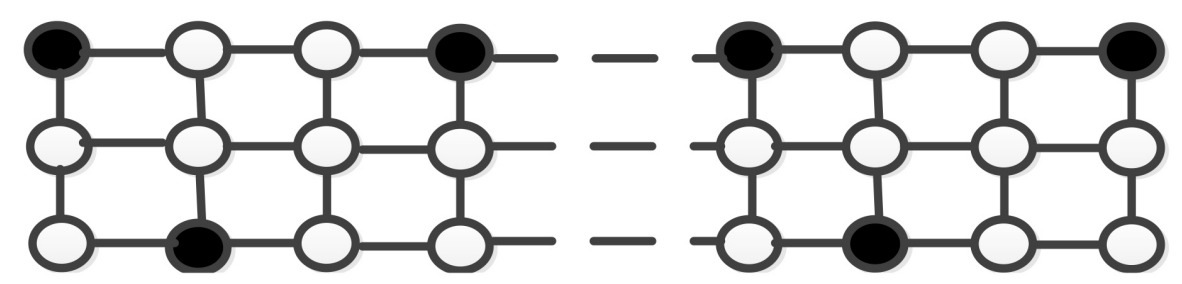

Figure 5. Case $n=1(\bmod 3)$.

\section{Case $n \equiv 2(\bmod 3)($ Figure 6$)$}

In this case we add to two columns to the graph so, the numeral to the vertices in the $B$ set at any two successive columns is less or equals 2 , so the signed domination number will increase of 2 than the signed domination number in case of $n \equiv 0(\bmod 3)$. If we add the vertices $(n, 2)$ and $(n-1,0)$ to $B$ set then $f$ remains a signed dominating function of the graph, and

$$
B=\left\{(0,3 j): 0 \leq j \leq\left\lfloor\frac{n-1}{3}\right\rfloor \cup(2,3 j+1): 0 \leq j \leq\left\lfloor\frac{n-2}{3}\right\rfloor\right\} \cup\{(0, n-1),(2, n)\} .
$$

Consequently, the domination number will be:

$$
\gamma_{s}\left(P_{3} \times P_{n}\right)=\frac{5(n-2)}{3}+2 .
$$

Theorem 2.2. Let $n$ be a positive integer:

$$
\begin{aligned}
& \text { If } n \neq 1(\bmod 4) \text {, then } \gamma_{s}\left(P_{4} \times P_{n}\right)=2 n ; \\
& \text { If } n=1(\bmod 4) \text {, then } \gamma_{s}\left(P_{4} \times P_{n}\right)=2 n-2 \text {. }
\end{aligned}
$$

Proof: Let $f$ be a signed domination function of the $\left(P_{4} \times P_{n}\right)$ graph. And let A, $B, K_{p} A_{j}$ and $B_{j}$ are the previously defined sets, Whatever $j$ is then $\left|B_{j}\right|+\left|B_{j+1}\right| \leq 2$, we notice that $B_{j} \leq 2$ so, discuss the following cases:

Case a. $\left|B_{j}\right|=2$. Then:

a.1. $K_{j} \cap B=\{(1, j),(4, j)\}$ or $K_{j} \cap B=\{(2, j),(3, j)\}$. 

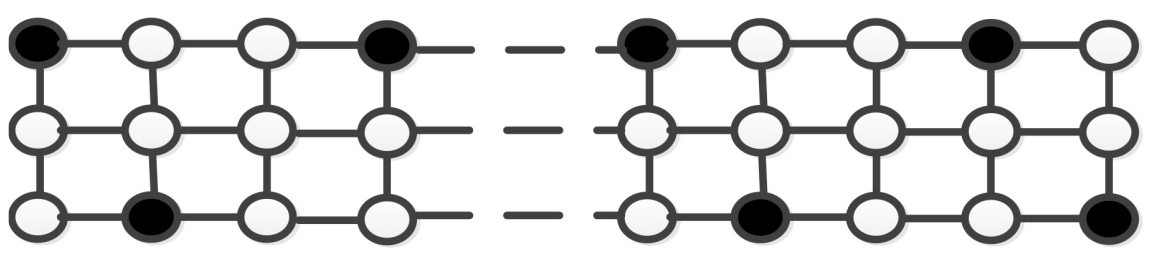

Figure 6. Case $n=2(\bmod 3)$.

In this case all the $j+1^{\text {th }}$ column vertices belong to the $A$ set vertices so, the two remained vertices of the $f^{\text {th }}$ column (Figure 7):

a.2. $K_{j} \cap B=\{(1, j),(3, j)\}$ or $K_{j} \cap B=\{(2, j),(4, j)\}$.

In this case one vertex at most $j+1^{\text {th }}$ or $j-1^{\text {th }}$ column vertices can belong to the $B$ set vertices, either $\left|B_{j}\right|+\left|B_{j+1}\right| \leq 2$ or $\left|B_{j-1}\right|+\left|B_{j}\right| \leq 2$ (Figure 8).

The vertices $(1, j),(2, j)$ can't belong to the $B$ set at the same time, neither the vertices $(3, j),(4, j)$ because both of the vertices $(1, j),(4, j)$ from the third degree and can't connect with any one of the $B$ set vertices.

Case b. $\left|B_{j}\right|=1$ : then we discuss the following cases:

b.1. If $(1, j) \in B$ or $(4, j) \in B$ then one of the $j+1^{\text {th }}$ column vertices at most can be from the $B$ set vertices (Figure 9).

b.2. If $(2, j) \in B$ or $(3, j) \in B$ then two of the $j+1^{\text {th }}$ column vertices at most can be from the $B$ set, and in this case all the $j+2^{\text {th }}$ column vertices are from the $A$ set vertices, and one vertex of the $j-1^{\text {th }}$ column vertices at most can belong to the $B$ set vertices, in this case $\left|B_{j+1}\right|+\left|B_{j+2}\right| \leq 2$ and also $\left|B_{j-1}\right|+\left|B_{j}\right| \leq 2$ (Figure 10).

In all previous cases we conclude that every two successive columns include two of the $B$ set vertices at most, then $\gamma_{s}\left(P_{4} \times P_{n}\right) \geq 2 n$. And the case of $(b-2)$ doesn't achieve in case of $[n \equiv 0(\bmod 4)]$ because if $(2, j) \in B$ in the $j-1^{\text {th }}$ column then $(2, j+1) \notin B$, as if $(3, j) \in B$ then $(3, j+1) \notin B$. so, in case of $n \equiv 0(\bmod 4)$ this will make $\gamma_{s}\left(P_{4} \times P_{n}\right) \geq 2 n$.

To find the upper bound of the signed domination number of $\left(P_{3} \times P_{n}\right)$ graph, let's define (Figure 11):

$$
B=\left\{(0,4 j),(3,4 j): 0 \leq j \leq\left\lfloor\frac{n-1}{4}\right\rfloor \cup(1,4 j+2),(2,4 j+2): 0 \leq j \leq\left\lfloor\frac{n-3}{4}\right\rfloor\right\} .
$$

We noticed that if the $B$ set vertices are the vertices which have the weight -1 of the $P_{4} \times P_{n}$ graph, every one of the graph vertices achieves the signed dominating function so, the signed domination number of the $P_{4} \times P_{n}$ graph will be: $\gamma_{s}\left(P_{4} \times P_{n}\right) \leq 4 n-2 n \leq 2 n$.

Consequently: $\gamma_{s}\left(P_{4} \times P_{n}\right)=2 n: n=0(\bmod 4)$.

Case $n \equiv 1(\bmod 4)$ (Figure 12$)$

If we add a column to the previous graph then the vertices $(0, n)$ and $(3, n)$ are of the $B$ set vertices, so:

$$
\begin{aligned}
B=\{ & \left.(0,4 j),(3,4 j): 0 \leq j \leq\left\lfloor\frac{n-1}{4}\right\rfloor \cup(1,4 j+2),(2,4 j+2): 0 \leq j \leq\left\lfloor\frac{n-3}{4}\right\rfloor\right\} \\
& \cup\{(0, n),(3, n)\} .
\end{aligned}
$$



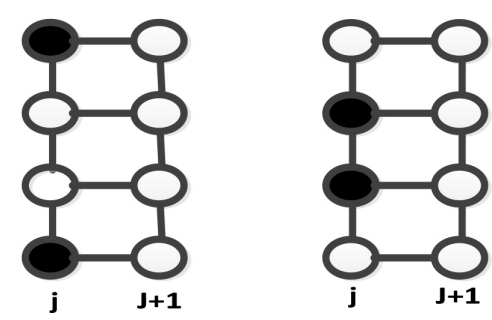

Figure 7. Case a.1.
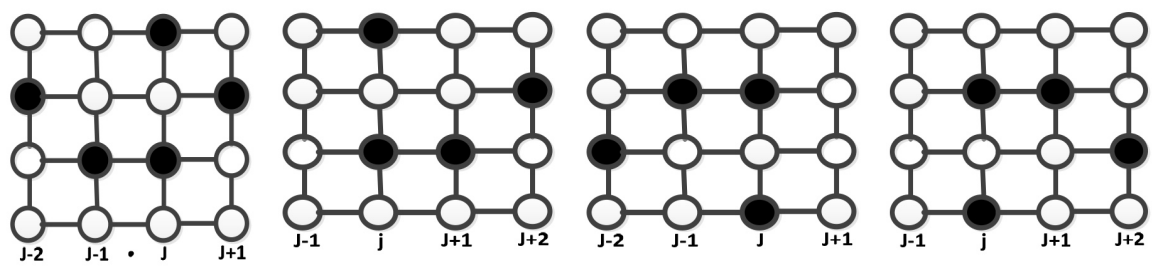

Figure 8. Case a.2.
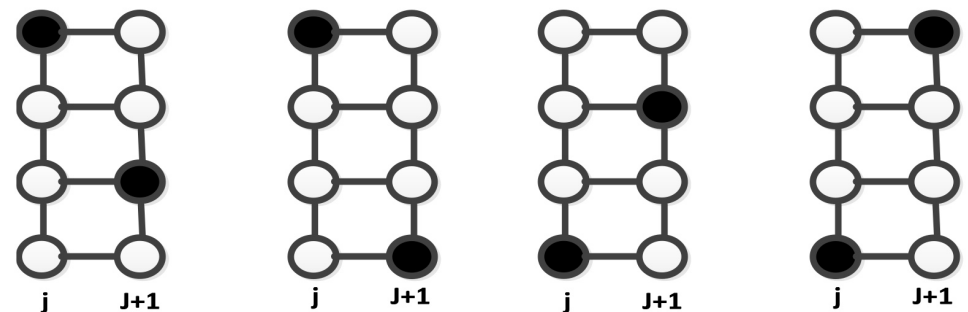

Figure 9. Case b.1.
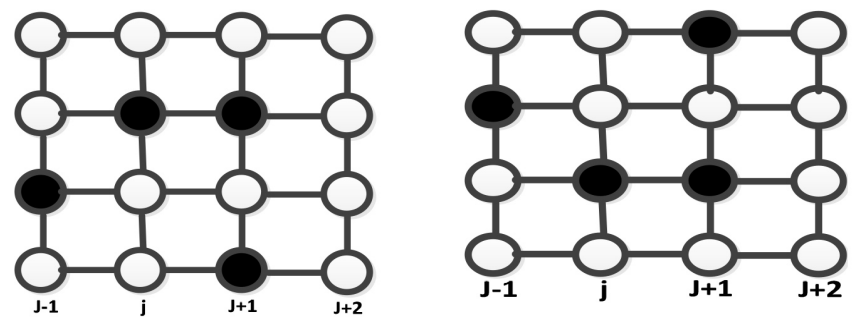

Figure 10. Case b.2.
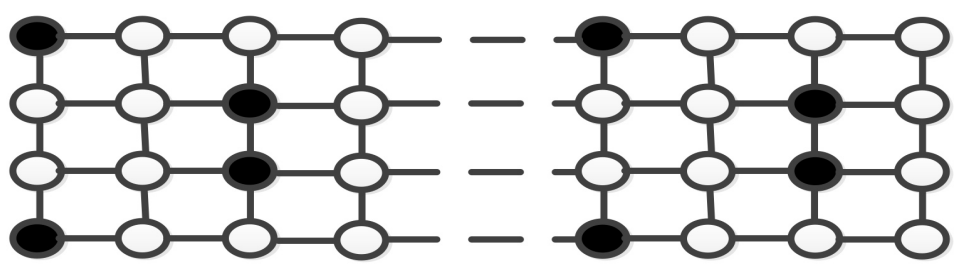

Figure 11. The B set.

The signed domination number at the last column will be equal to zero, then number of the columns will increase of 1 , without any increment for the signed domination number then $\gamma_{s}\left(P_{4} \times P_{n}\right)=2(n-1)=2 n-2$.

Consequently: $\gamma_{s}\left(P_{4} \times P_{n}\right)=2 n-2: n=1(\bmod 4)$. 

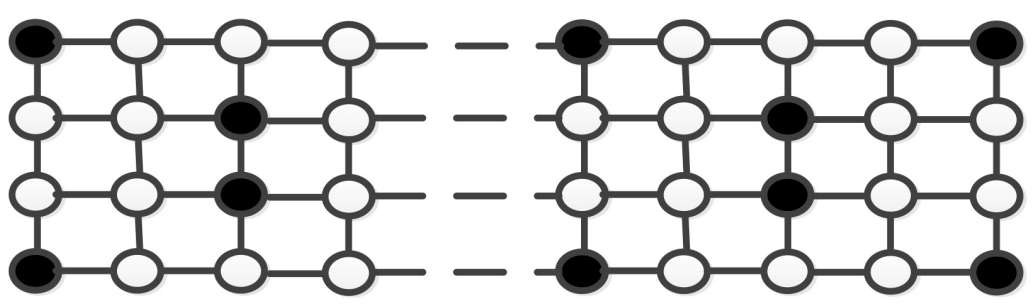

Figure 12. Case $n=1(\bmod 4)$.

\section{Case $n \equiv 2(\bmod 4)$}

In this case we add to two columns of the graph, then we notice that the last column doesn't include any one of the $B$ set vertices so, the signed domination number then:

$$
\gamma_{s}\left(P_{4} \times P_{n}\right)=2 n: n \equiv 2(\bmod 4) .
$$

\section{Case $n \equiv 3(\bmod 4)$}

In this case when we add to three columns of the graph, then we notice that only one of the vertices $(3, n)$ and $(2, n)$ is from the $B$ set. So, the signed domination number then:

$$
\begin{array}{ll} 
& \gamma_{s}\left(P_{4} \times P_{n}\right)=2 n: n \equiv 3(\bmod 4) . \\
\text { Consequently: } & \gamma_{s}\left(P_{4} \times P_{n}\right)=2 n: n \neq 1(\bmod 4) \\
& \gamma_{s}\left(P_{4} \times P_{n}\right)=2 n-2: n=1(\bmod 4) .
\end{array}
$$

Theorem 2.3. Let $n$ be a positive integer, for $n \geq 5$ then

$$
\begin{aligned}
& \text { If } n=0(\bmod 5) \text {, then } \gamma_{s}\left(P_{5} \times P_{n}\right)=\frac{9 n}{5}+2 ; \\
& \text { If } n=2,4(\bmod 5) \text {, then } \gamma_{s}\left(P_{5} \times P_{n}\right)=\frac{9 n}{5}+3 \text {; } \\
& \text { If } n=1,3(\bmod 5) \text {, then } \gamma_{s}\left(P_{5} \times P_{n}\right)=\frac{9 n}{5}+4 \text {. }
\end{aligned}
$$

Proof: Let $f$ be a signed domination function of the $P_{5} \times P_{n}$ graph. And $A, B$, $K_{p} A_{j}$ and $B_{j}$ are the previously defined sets, then whatever $1 \leq j \leq n-4$, then: $\sum_{i=j}^{j+4}\left|B_{i}\right| \leq 8$. We discuss the following cases:

\section{Case a. $\left|B_{j}\right|=3$ (Figure 13)}

a.1. If $(1, j),(3, j)$ and $(5, j) \in B$ then $(3, j+1) \in \mathrm{B}$ and one of the vertices $(2, j+2)$ or $(4, j+2)$ is of the $B$ set vertices and in the two cases $(2, j+3)$ and $(4$, $j+3)$ are of the B set vertices and only one vertex of the $j+4^{\text {th }}$ column.

a.2. If $(1, j),(3, j)$ and $(4, j) \in B$ then the $j+1^{\text {th }}$ column doesn't include any one of the $B$ set vertices. The $j+2^{\text {th }}$ column include three of the $B$ set vertices, the $j+3^{\text {th }}$ column doesn't include any one of the $B$ set vertices. Then every two successive columns include three vertices of the $B$ set. And every ten successive columns include fifteen vertices of the $B$ set.

Case b. $\left|B_{j}\right|=2$ :

b.1. If $(1, j)$ and $(3, j) \in B$ then $(3, j+1)$ and $(5, j+1) \in B,(2, j+2) \in B$ and also $(2, j+3)$ and $(4, j+3)$ are of the B set vertices, And the $j+4^{\text {th }}$ column include only the vertex $(4, j+4)$. 

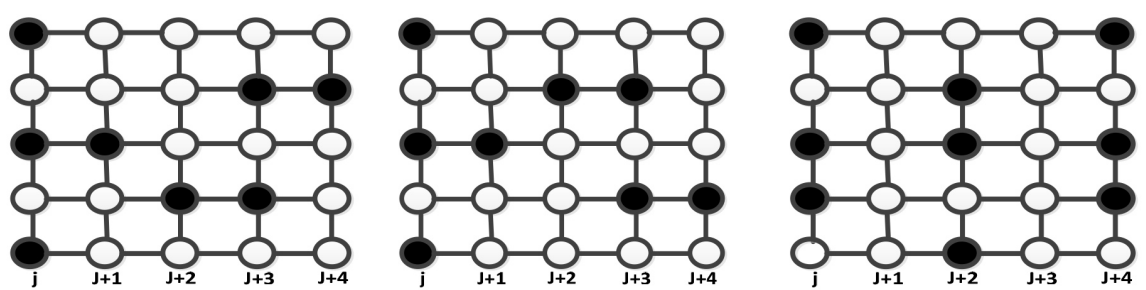

Figure 13. Case a.

b.2. If $(1, j)$ and $(4, j) \in B$ then $(3, j+1) \in B,(2, j+2)$ and $(5, j+2) \in B$, $(2, j$ $+3) \in B,(3, j+4)$ and $(4, j+4) \in B$.

b.3. If $(1, j)$ and $(5, j) \in B$ then $(3, j+1) \in B$. And the $j+2^{\text {th }}$ column include one vertex of the $B$ set vertices at most. And the $j+3^{\text {th }}$ column include two vertices at most. And the $j+4^{\text {th }}$ column include only one vertex.

b.4. If $(2, j)$ and $(3, j) \in B$ then $(4, j+1)$ or $(5, j+1) \in B$, and the $j+2^{\text {th }}$ column include two vertices of the $B$ set vertices at most, And the $j+3^{\text {th }}$ column include one vertex at most. And the $j+4^{\text {th }}$ column include only two vertices.

b.5. If $(2, j)$ and $(4, j) \in B$ then $(2, j+1),(4, j+1) \in B$. And the $j+2^{\text {th }}$ column doesn't include any one of the $B$ set vertices $(1, j+3),(3, j+3),(5, j+3) \in B$, and $(3, j+4) \in B$, Then if $\left|B_{j}\right|=2$ then: $\sum_{i=j}^{j+4}\left|B_{j}\right| \leq 8 \quad$ (Figure 14).

Case c. $\left|B_{j}\right|=1$ (Figure 15)

c.1. If $(1, j) \in B$ or $(2, j) \in B$ or $(4, j) \in B$ or $(5, j) \in B$ then the $j+1^{\text {th }}$ column include two of the $B$ set vertices and the $j+2^{\text {th }}$ column include one vertex at most. And the $j+3^{\text {th }}$ column include two of the $B$ set vertices at most. In this case the $j+4^{\text {th }}$ column include one of the $B$ set vertices at most.

c.2. If $(3, j) \in B$ then $(1, j+1) \in B,(3, j+1) \in B,(5, j+1) \in B$. And the $j+2^{\text {th }}$ column doesn't include any one of the $B$ set vertices. And the vertices $(2, j+3)$, $(4, j+3),(2, j+4)$ and $(4, j+4)$ belong to the $B$ set vertices and the other cases are repeated.

Whatever $1 \leq j \leq n-4$, then $\sum_{i=j}^{j+4}\left|B_{i}\right| \leq 8$.

All of the five successive columns include eight of the $B$ set vertices, then:

$$
\gamma_{s}\left(P_{5} \times P_{n}\right) \geq 5 n-2\left(\frac{8 n}{5}\right) \Rightarrow \gamma_{s}\left(P_{5} \times P_{n}\right) \geq 5 n-\frac{16 n}{5} \Rightarrow \gamma_{s}\left(P_{5} \times P_{n}\right) \geq \frac{9 n}{5} \text {. }
$$

Let's defined:

$$
\begin{aligned}
B=\{ & (1,5 j+1),(3,5 j+1): 0 \leq j \leq\left\lfloor\frac{n-1}{5}\right\rfloor \cup(3,5 j+2) \\
& \left.\cup(5,5 j+2): 0 \leq j \leq\left\lfloor\frac{n-2}{5}\right\rfloor\right\} \cup\left\{(2,5 j+3): 0 \leq j \leq\left\lfloor\frac{n-3}{5}\right\rfloor\right. \\
& \cup(2,5 j+4),(4,5 j+4): 0 \leq j \leq\left\lfloor\frac{n-4}{5}\right\rfloor \\
& \left.\cup(4,5 j+5): 0 \leq j \leq\left\lfloor\frac{n-5}{5}\right\rfloor\right\}
\end{aligned}
$$

then $|B|=\frac{8 n}{5} \quad$ (Figure 16). 

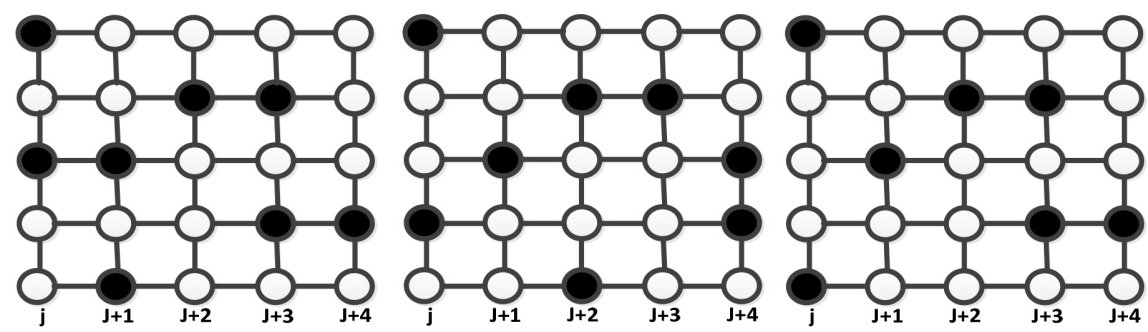

Figure 14. Case b.
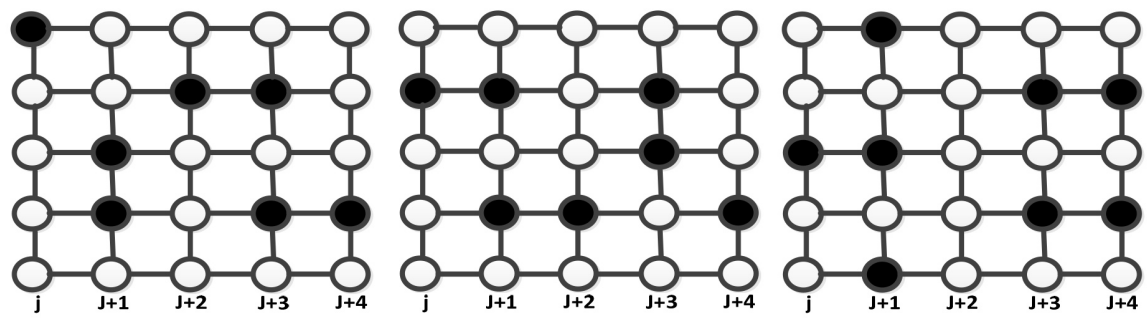

Figure 15. Case c.

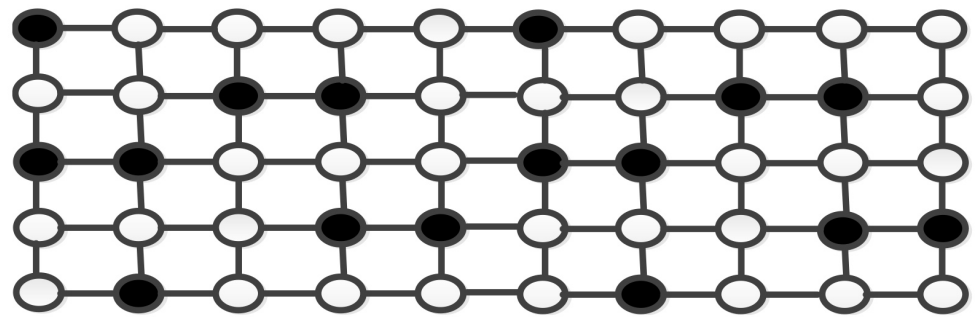

Figure 16. The B set.

We noticed that if the $B$ set vertices are the vertices which have the weight -1 of the graph $P_{5} \times P_{n}$ then every one of the graph vertices achieve the signed domination function then the signed domination number of the $P_{5} \times P_{n}$ graph will be:

$$
\gamma_{s}\left(P_{5} \times P_{n}\right) \leq 5 n-2\left(\frac{8 n}{5}\right) \text { then } \gamma_{s}\left(P_{5} \times P_{n}\right) \leq \frac{9 n}{5} .
$$

But it proves easily, that the first and second columns of $P_{5} \times P_{n}$, have at most three vertices of the $B$ set vertices. As well the vertices $(1,1)$ and $(3,1)$ they cannot belong to set $B$ in the same time, then if we delete the vertex $(3,1)$ of $B$ set, then the signed domination number of the $P_{5} \times P_{n}$ will be: $\gamma_{s}\left(P_{5} \times P_{n}\right)=\frac{9 n}{5}+2$.

Case $\boldsymbol{n} \equiv \mathbf{0}(\bmod 5)$ (Figure 17)

If we add three columns at the beginning, two columns at the end and add the vertices $(1,1),(5,1),(3,2),(4,3),(2, n-1),(3, n-1)$ and $(5, n)$. So, $n \equiv 0(\bmod 5)$, and $\gamma_{s}\left(P_{5} \times P_{n}\right)=\frac{9 n}{5}+2$.

Case $n \equiv 2(\bmod 5)($ Figure 18$)$

Note that the last two columns contain three vertices of $B$ set vertices, such the signed domination number in these two columns equals 4 , then: 


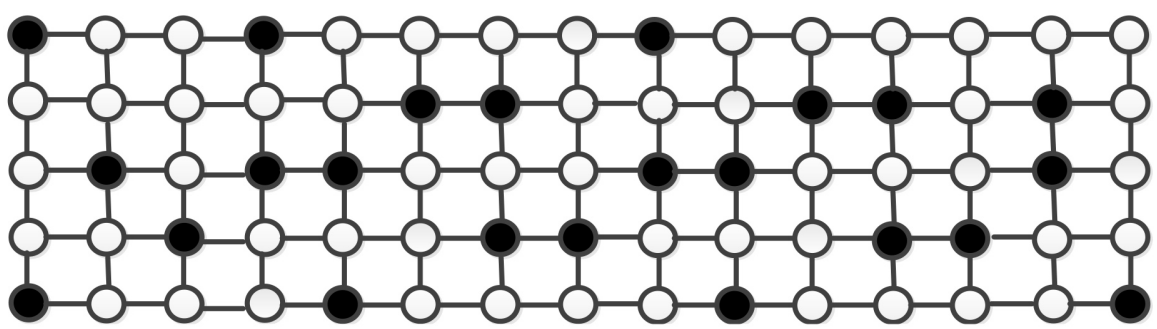

Figure 17. Case $n=0(\bmod 5)$.
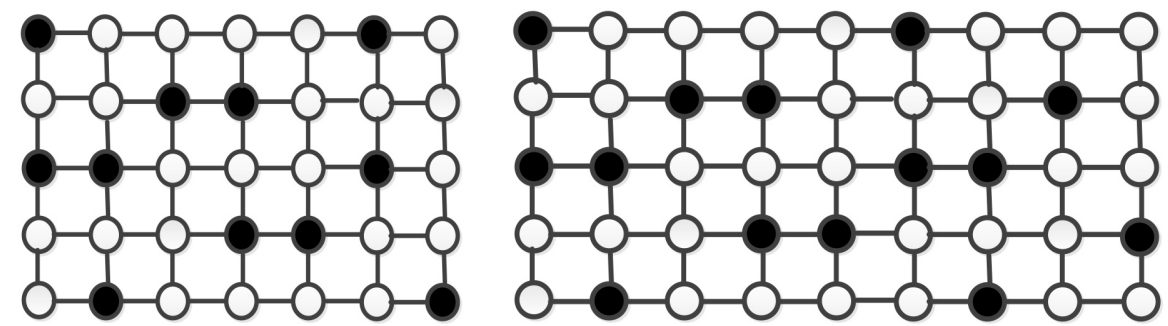

Figure 18. Case $n=2,4(\bmod 5)$.
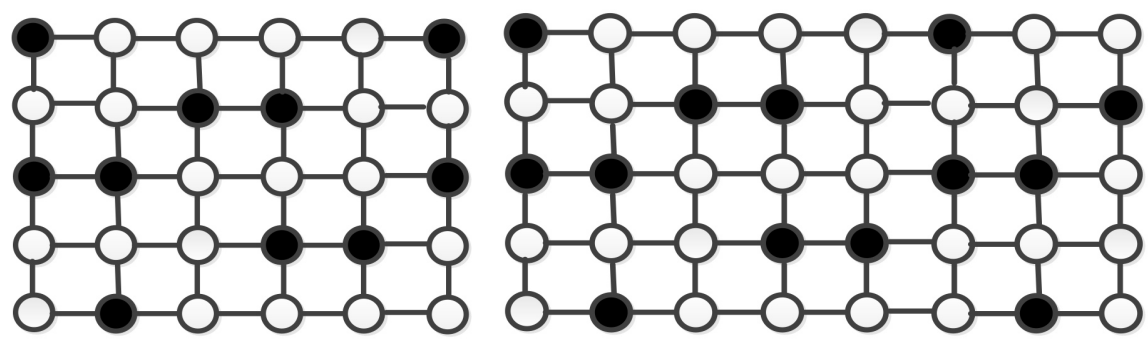

Figure 19. Case $n=1,3(\bmod 5)$.

$$
\gamma_{s}\left(P_{5} \times P_{n}\right)=\frac{9(n-2)}{5}+2+4=\frac{9 n}{5}+\frac{12}{5}=\frac{9 n}{5}+3 .
$$

\section{Case $n \equiv 4(\bmod 5)$}

Note that the last four columns contain six vertices of $B$ set vertices, such the signed domination number in these columns equals 8 , then:

$$
\gamma_{s}\left(P_{5} \times P_{n}\right)=\frac{9(n-4)}{5}+2+8=\frac{9 n}{5}+\frac{14}{5}=\frac{9 n}{5}+3 .
$$

Consequently: $\gamma_{s}\left(P_{5} \times P_{n}\right)=\frac{9 n}{5}+3: n=2,4(\bmod 5)$.

Case $n \equiv 1,3(\bmod 5)$ (Figure 19$)$

In this case we note that when you delete one vertex of the $B$ set vertices previously defined of the last column, and the signed domination number is increasing by 2 in both cases then:

$$
\gamma_{s}\left(P_{5} \times P_{n}\right)=\frac{9 n}{5}+4 .
$$

Consequently: $\gamma_{s}\left(P_{5} \times P_{n}\right)=\frac{9 n}{5}+4: n=1,3(\bmod 5)$. 


\section{Conclusion}

In this paper, we studied The signed domination numbers of the Cartesian product of two paths $P_{m}$ and $P_{n}$ for $m=3,4,5$ and arbitrary $n$. we will work to find the signed domination numbers of the Cartesian product of two paths $P_{m}$ and $P_{n}$ for arbitraries $m$ and $n$.

\section{Conflicts of Interest}

The authors declare no conflicts of interest regarding the publication of this paper.

\section{References}

[1] Dunbar, J., Hedetniemi, S.T., Henning, M.A. and Slater, P.J. (1995) Signed Domination in Graphs. In: Graph Theory, Combinatorics and Applications, John Wiley \& Sons, New York, 311-322.

[2] Broere, I., Hattingh, J.H., Henning, M.A. and McRae, A.A. (1995) Majority Domination in Graphs. Discrete Mathematics, 138, 125-135. https://doi.org/10.1016/0012-365X(94)00194-N

[3] Cockayne, E.J. and Mynhardt, C.M. (1996) On a Generalization of Signed Dominating Functions of Graphs. Ars Combinatoria, 43, 235-245.

[4] Favaron, O. (1996) Signed Domination in Regular Graphs. Discrete Mathematics, 158, 287-293. https://doi.org/10.1016/0012-365X(96)00026-X

[5] Haasa, R. and Wexlerb, T.B. (2004) Signed Domination Numbers of a Graph and Its Complement. Discrete Mathematics, 283, 87-92. https://doi.org/10.1016/j.disc.2004.01.007

[6] Hosseini, S.M. (2015) New Bounds on the Signed Domination Numbers of Graphs. Australasian Journal of Combinatorics, 61, 273-280. 